\title{
Short-cut method for assessing solvents for gas cleaning by reactive absorption
}

\author{
Dan Vasiliu, Elmar Kessler, Erik von Harbou, and Hans Hasse \\ Laboratory of Engineering Thermodynamics (LTD), University of \\ Kaiserslautern, 67663 Kaiserslautern, Germany
}

May 22, 2020

\section{Abstract}

A new short-cut method (NoVa) for assessing solvents for gas cleaning by reactive absorption is presented. It considers the absorption / desorption cycle using the assumption of infinite number of stages in both columns. For a given feed and removal rate, the method yields an estimate for the specific regeneration energy $q$ as a function of the solvent circulation rate $L / G$. The sole solventdependent input consists of two correlations describing the gas solubility at absorber and desorber conditions and estimates of caloric properties. Furthermore, a simple equation (SolSOFT) for correlating the gas solubility as a function of the gas loading of the solvent is presented. A theoretical analysis of the process reveals general properties of the dependency of $q$ on $L / G$. The NoVa method is described and tested using amine-based solvents for post combustion carbon capture as examples.

Core topic area: Separation processes - Absorption science and technology

Keywords: absorption-desorption process, short-cut method, solvent screening, gas solubility, correlation 


\section{Introduction}

Absorption / desorption processes are widely used for gas cleaning. For assessing solvents for such processes in the screening phase, short-cut methods are often used, which need only a limited amount of input data on the process and the solvent. Designing short-cut methods for assessing reactive solvents for absorption / desorption processes is particularly challenging due to the strongly non-linear nature of the gas solubility as a function of the loading. Several approaches to tackle

these challenges have been described in the literature. The methods of Notz et al., $\underline{1}$ Kim et al., $\underline{2}$ Jiang et al. $\underline{3}$ and Knuutila et al. $\stackrel{4}{ }$ are among the most prominent ones. All of them aim at estimating the specific regeneration energy for a given solvent flowrate. The method of Notz et al. $\frac{1}{=}$ stands out as it is based on a well-defined simple physical model of the full absorption / desorption process and is therefore used as a basis for the present work.

Notz et al. $\stackrel{1}{-}$ have developed their method as part of work on screening solvents for post combustion carbon capture (PCC). In their method, Notz et al. $\stackrel{1}{-}$ specify the gaseous feed to the process and the desired removal rate. The method then yields the regeneration energy as a function of the solvent circulation rate for the chosen conditions. That function typically has a minimum, so that the minimum regeneration energy and the corresponding solvent circulation rate can be determined. The procedure is repeated for different solvents and the resulting numbers for the minimum regeneration energy and the corresponding solvent circulation rate are compared to obtain a ranking of the solvents. The primary goal of the method is obtaining that ranking. It has been shown, however, that the method not only succeeds in this but also yields results for the minimum regeneration energy that agree with experimental pilot plant data and simulation results obtained with more sophisticated methods. $1: \underline{5}$ The only data on the solvent needed for the application of the method of Notz et al. $\underline{\underline{1}}$ is gas solubility data at absorber and desorber conditions (for PCC solvents, typically isothermal data at $313 \mathrm{~K}$ and $393 \mathrm{~K}$ are used) and some crude estimates for caloric properties (solvent heat capacity, enthalpy of absorption).

However, the method of Notz et al. $\frac{1}{}$ also has some drawbacks: first, the equilibrium curve (partial pressure of gas versus solvent loading) is used in a discretized form. The discretization 
is not only tedious, it also induces arbitrariness in the results and it may lead to artifacts. In the present work, therefore, a continuous representation of the equilibrium curve is used and a new equation is presented that is particularly suited for the correlation of equilibrium curves of the gas solubility in reactive solvents. It is called SolSOFT (from solubility soft model). Second, the method of Notz et al. $\underline{\underline{1}}$ uses a fixed number of stages both for the absorber and desorber. The choice of the number of stages is again somehow arbitrary. We prefer to use the assumption of infinite number of stages in both columns which has been shown to be very useful for conceptual design of distillation processes. $\underline{6} 7 ; 8 ; 9$ Removing the arbitrariness establishes safer ground for comparisons and yields the possibility to draw general conclusions. Based on these assumptions, a new shortcut method was developed. It is called NoVa (from Notz \& Vasiliu). The SolSOFT equation and the NoVa short-cut method are thoroughly discussed for the first time in this work. Nevertheless, they have already been employed in previous studies for evaluation of solvents for carbon capture (e.g., Vasiliu et al., 10 Kessler et al. $\stackrel{11}{ })$.

The remainder of this paper is organized as follows: In Section 3, we describe the NoVa method and give all equations. After an overview, the models for the absorber and desorber are explained. They are equilibrium stage models based on the assumption of an infinite number of stages in each of the two columns. Different cases of their operation are identified, which form a basis for the process analysis. In the last part of Section 3, the enthalpy model is described. The process model presented in Section 3 is based on the knowledge of the gas solubility isotherms at the absorber and desorber conditions. The SolSOFT equation, which is suitable for the description of such isotherms, is presented in Section 4. In Section 5, examples for the application of the NoVa method are presented. A novel solvent is compared to two well-known ones. It is emphasized that the main point here is not the comparison of the three solvents but the illustration of how the new NoVa short-cut method works and which type of results are obtained. The new amine is butyltriacetonediamine (BuTAD), and has recently been studied by our group. 10 The reference amines are monoethanolamine (MEA) and methyldiethanolamine (MDEA). The solvents were aqueous solutions with $0.3 \mathrm{~g} / \mathrm{g}$ amine. Conclusions are drawn in Section 6. 


\section{NoVa short-cut method}

Figure 1 shows the flow sheet considered in the NoVa method. It also introduces the notations used in the present work. The absorption of $\mathrm{CO}_{2}$ in reactive solvents like aqueous amine solutions, as commonly employed in PCC applications, is used here as an example for describing the NoVa method. The idea behind NoVa is, however, generally applicable for absorption-desorption processes single gases, since its premises do not contain anything inherent that confines its application to $\mathrm{CO}_{2}$ capture. In the absorber, the $\mathrm{CO}_{2}$ from the rich gas is absorbed exothermically into the lean solvent in a counter-current washing process. The rich gas has a mass flowrate $\dot{m}_{\mathrm{G}}^{\mathrm{Abs}}$, a molar mass $M_{\mathrm{G}}^{\mathrm{Abs}}$ and the mole fraction of $\mathrm{CO}_{2}$ is $x_{\mathrm{CO}_{2}}^{\mathrm{Abs} \text {,in }}$. The mass flowrate of the unloaded (without $\mathrm{CO}_{2}$ ) lean solvent is $\dot{m}_{\mathrm{L}}^{\mathrm{Abs}}$. The lean gas leaves the plant at the top of the absorber and the rich solvent goes from the bottom of the absorber to the desorber. Such an absorber operates typically at a temperature of around $T^{\mathrm{Abs}} \approx 313 \mathrm{~K}$ and at a total pressure of around $p^{\mathrm{Abs}} \approx 1 \mathrm{bar}$. In the desorber, the rich solvent releases $\mathrm{CO}_{2}$ in an endothermic process. The desorber reboiler duty $\dot{Q}^{\mathrm{Reb}}$ is the main contribution to the total energy demand of the process and it is therefore the main target for optimization. The lean solvent goes from the bottom of the reboiler back to the absorber. The solvent-saturated $\mathrm{CO}_{2}$ leaving the desorber passes first a condenser before it is sent to its destination (e.g. sequestration, further processing). The mass flowrate of the recovered $\mathrm{CO}_{2}$ is $\dot{m}_{\mathrm{CO}_{2}}^{\text {Des,out }}$. The condensate contains mostly water and is fed back at the top of the desorber. The desorber operates typically at a total pressure of around $p^{\text {Des }} \approx 2$ bar, which corresponds to a temperature of around $T^{\text {Des }} \approx 393 \mathrm{~K}$. A regeneration heat exchanger, in which the cold rich solvent is heated up by the hot lean solvent, is used for thermal integration. After the regeneration heat exchanger, the lean solvent passes a cooler before entering the absorber.

The three parameters of this process that are most relevant for NoVa are: the removal rate $\psi$

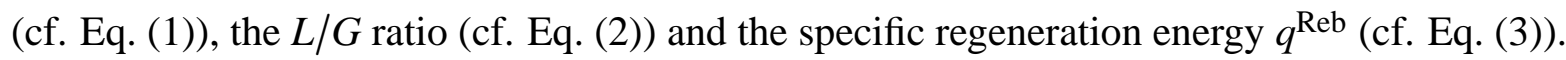

$$
\psi=\frac{\dot{m}_{\mathrm{CO}}^{\text {Des,out }}}{\dot{m}_{\mathrm{CO}_{2}}^{\mathrm{Abs}, \text { in }}}=\frac{1}{x_{\mathrm{CO}_{2}}^{\mathrm{Abs}, \text { in }}} \cdot \frac{M_{\mathrm{G}}^{\mathrm{Abs}}}{\dot{m}_{\mathrm{G}}^{\mathrm{Abs}}} \cdot \frac{\dot{m}_{\mathrm{CO}_{2}}^{\text {Des,out }}}{M_{\mathrm{CO}_{2}}}
$$




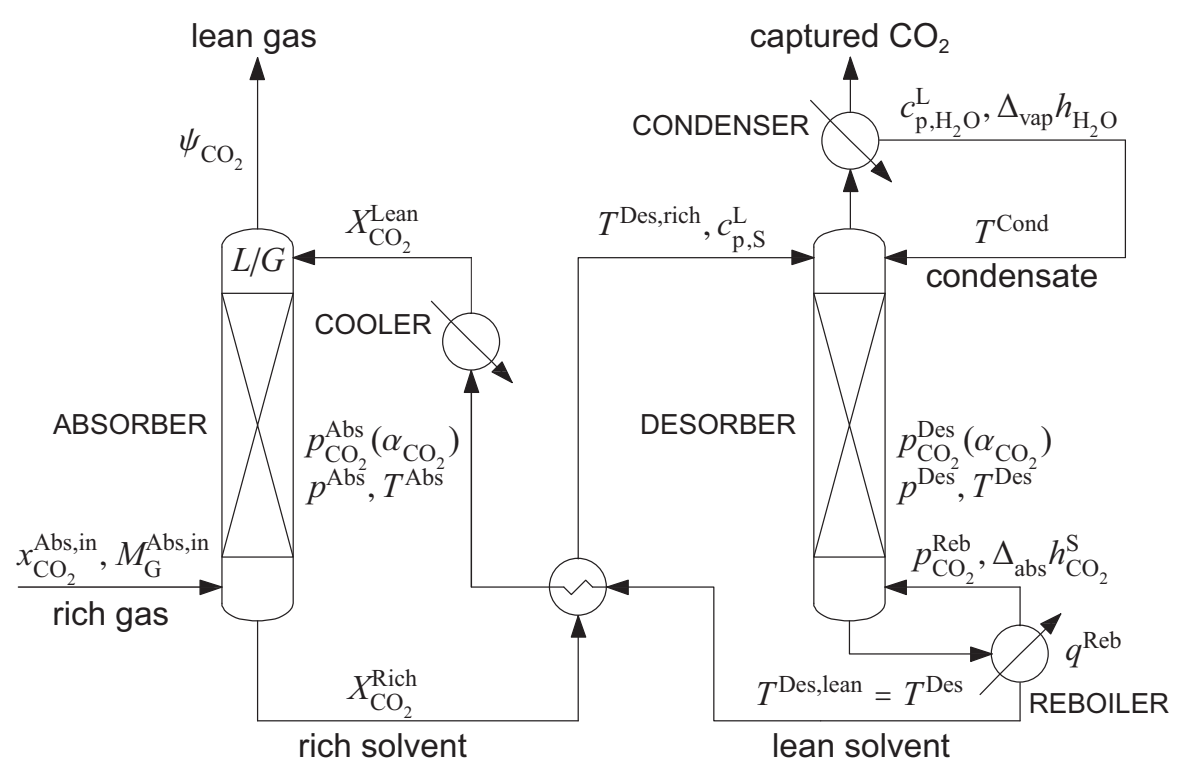

Figure 1: Simplified flowsheet of a typical absorption / desorption process for $\mathrm{CO}_{2}$-capture and nomenclature used in the present work.

$$
\begin{gathered}
L / G=\frac{\dot{m}_{\mathrm{L}}^{\mathrm{Abs}}}{\dot{m}_{\mathrm{G}}^{\mathrm{Abs}}} \\
q^{\mathrm{Reb}}=\frac{\dot{Q}^{\mathrm{Reb}}}{\dot{m}_{\mathrm{CO}_{2}}^{\text {Des,out }}}
\end{gathered}
$$

The $L / G$ ratio is the conventional variable commonly used in the literature. It is convenient for the following to work with the variables $\xi$ and $\tilde{\xi}$ instead of $L / G$, cf. Eq. (4) and Eq. (5), respectively. Using $\xi$ instead of $L / G$ highlights important properties of the NoVa model and $\tilde{\xi}$ is used for brevity and clarity of the presentation of the mathematical model.

$$
\begin{gathered}
\xi=\frac{L / G}{\psi} \\
\tilde{\xi}=\frac{M_{\mathrm{G}}^{\mathrm{Abs}}}{x_{\mathrm{CO}_{2}}^{\mathrm{Abs}, \mathrm{in}}} \cdot \xi
\end{gathered}
$$

NoVa is based on the following three premises: (I) both the absorber and the desorber are 
described by the equilibrium stage model with an infinite number of stages; (II) constant flowrates of gas and liquid and constant thermophysical properties are assumed for both columns; (III) the equilibrium curves are described by positive, monotonously increasing, convex and differentiable non-linear algebraic functions.

The basic run of NoVa consists of three consecutive steps: (I) absorber step, (II) desorber step, and (III) energy balance step. The procedure is described here using molalities $X_{i}$ for quantifying the loading of the solvent with the component $i$, i.e. mole number of component $i$ per kilogram of unloaded solvent (labeled $\mathrm{S}^{*}$ here). Here, $i=\mathrm{CO}_{2}$. In the literature, the loading is also expressed in moles $i$ per mole reactive component A (e.g. amine) contained in the solvent and denoted by $\alpha_{i}$. The relation between $X_{i}$ and $\alpha_{i}$ is given in Eq. (6).

$$
X_{i}=1000 \frac{x_{\mathrm{A}}^{(\mathrm{m}), \mathrm{S}^{*}}}{M_{\mathrm{A}}} \alpha_{i}
$$

$M_{\mathrm{A}}$ is the molar mass of $\mathrm{A}$ and $x_{\mathrm{A}}^{(\mathrm{m}), \mathrm{S}^{*}}$ is the mass fraction of $\mathrm{A}$ in the unloaded solvent $\mathrm{S}^{*}$.

In applying NoVa for assessing solvents, the basic run of NoVa is repeated for different numbers of $L / G$, holding all other input parameters constant. In the following, the calculations carried out in the basic run are described. The input and output of the basic run are summarized in Figure 2, It is assumed that the equilibrium curve as a function of the loading $\left(p_{\mathrm{CO}_{2}}^{\mathrm{Eq}}\left(X_{\mathrm{CO}_{2}}\right)\right)$ is strictly increasing and convex, which is regularly the case in reactive absorption. In the mathematical discussion, also the inverse function $X_{\mathrm{CO}_{2}}^{\mathrm{Eq}}\left(p_{\mathrm{CO}_{2}}\right)$ is used. The input and output of NoVa is summarized in Figure 2 and a flowchart showing its main steps is given in Supplementary Information A. For the nomenclature see also Figure 1. 


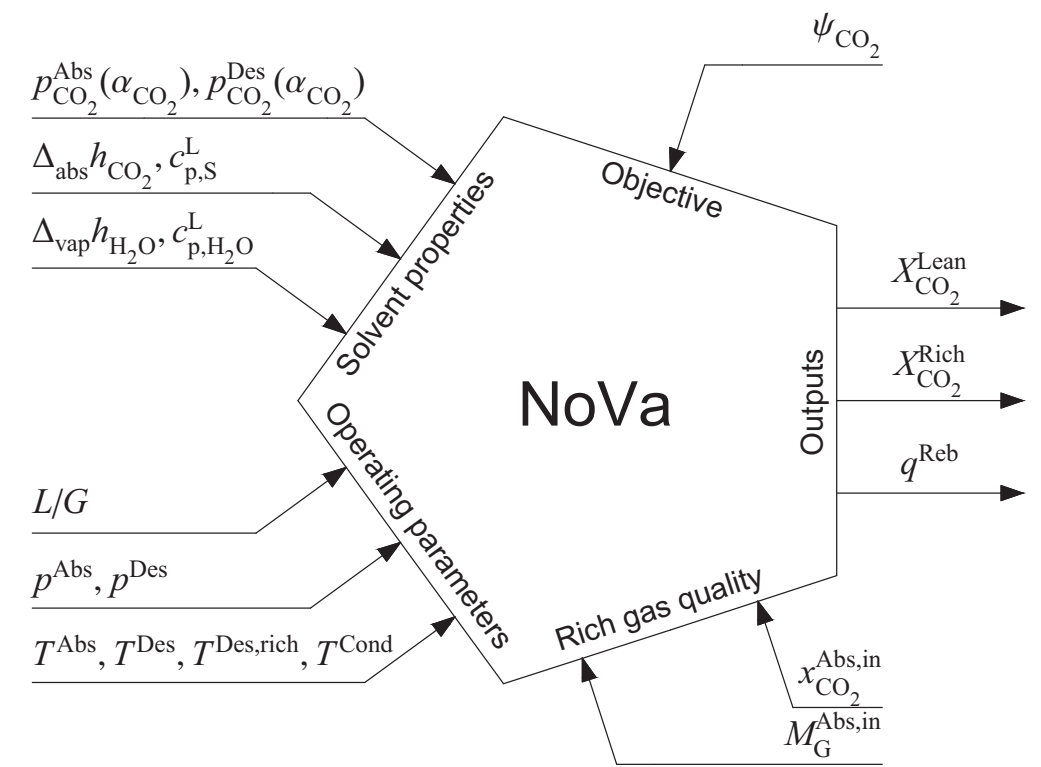

Figure 2: Input / output diagram of the basic run of the NoVa short-cut method. 


\subsection{Absorber step}

The partial pressure of $\mathrm{CO}_{2}$ at the inlet $\left(p_{\mathrm{CO}_{2}}^{\mathrm{Abs}, \mathrm{in}}\right)$ and at the outlet $\left(p_{\mathrm{CO}_{2}}^{\mathrm{Abs} \text { out }}\right)$ of the absorber is calculated with Eq. (7) and Eq. (8), respectively.

$$
\begin{gathered}
p_{\mathrm{CO}_{2}}^{\mathrm{Abs}, \text { in }}=x_{\mathrm{CO}_{2}}^{\mathrm{Abs} \text {,in }} p^{\mathrm{Abs}} \\
p_{\mathrm{CO}_{2}}^{\mathrm{Abs}, \text { out }}=(1-\psi) p_{\mathrm{CO}_{2}}^{\mathrm{Abs}, \text { in }}
\end{gathered}
$$

To meet the requirements of the three premises of NoVa two geometric conditions must be fulfilled in the McCabe-Thiele plot for the absorber: (1) the operating line and the equilibrium curve must have at least one pinch point (point of intersection); (2) all other points of the operating line must lie above the equilibrium curve. The three geometrically conceivable cases are shown in Figure 3. In case A1 (cf. Figure 3a), there is one pinch point at the top end of the operating line. In case A2 (cf. Figure $3 \mathrm{~b}$ ), there are two pinch points at the two ends of the operating line. In case A3 (cf. Figure $3 \mathrm{c}$ ), there is one pinch point at the bottom end of the operating line. All three cases (A1, A2 and A3) are feasible and are addressed by the algorithm described below.

Which of the three cases described above applies, depends on the value $\tilde{\xi}_{\text {crit }}^{\text {Abs }}$ as defined by Eq. (9). Depending on which case applies, the concentrations of $\mathrm{CO}_{2}$ in the lean $\left(X_{\mathrm{CO}_{2}}^{\mathrm{Lean}}\right)$ and the rich solvent $\left(X_{\mathrm{CO}_{2}}^{\mathrm{Rich}}\right)$ are calculated differently.

$$
\tilde{\xi}_{\mathrm{crit}}^{\mathrm{Abs}}=\frac{1}{X_{\mathrm{CO}_{2}}^{\mathrm{Abs}, \mathrm{eq}}\left(p_{\mathrm{CO}_{2}}^{\mathrm{Abs}, \mathrm{in}}\right)-X_{\mathrm{CO}_{2}}^{\mathrm{Abs}, \mathrm{eq}}\left(p_{\mathrm{CO}_{2}}^{\mathrm{Abs}, \text { out }}\right)}
$$

$\tilde{\xi}$ cannot take values smaller than $\tilde{\xi}_{\min }$, which is calculated with Eq. (10). At $\tilde{\xi}=\tilde{\xi}_{\min }$ the lean solvent is completely unloaded, i.e. $X_{\mathrm{CO}_{2}}^{\mathrm{Lean}}=0$ and the regeneration energy would be infinite $\left(q^{\operatorname{Reb}} \rightarrow \infty\right)$

$$
\tilde{\xi}_{\min }=\frac{1}{X_{\mathrm{CO}_{2}}^{\mathrm{Abs}, \mathrm{eq}}\left(p_{\mathrm{CO}_{2}}^{\mathrm{Abs}, \mathrm{in}}\right)}
$$




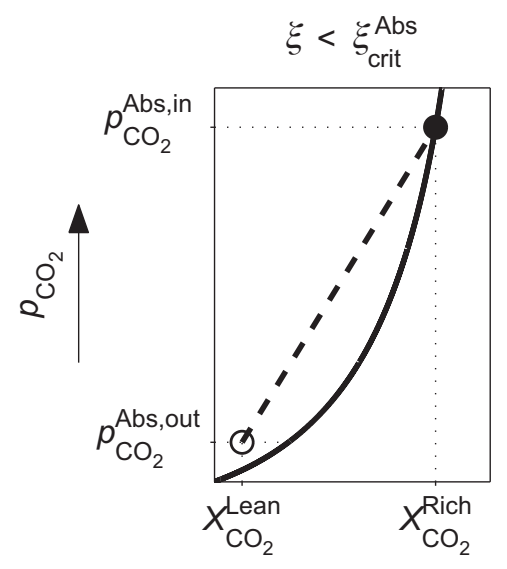

(a)

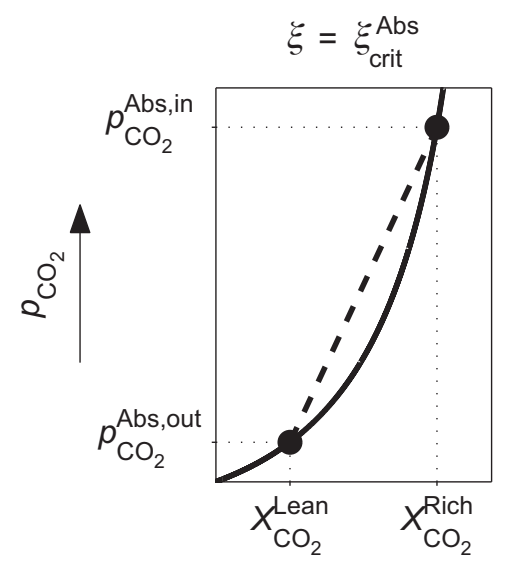

(b)

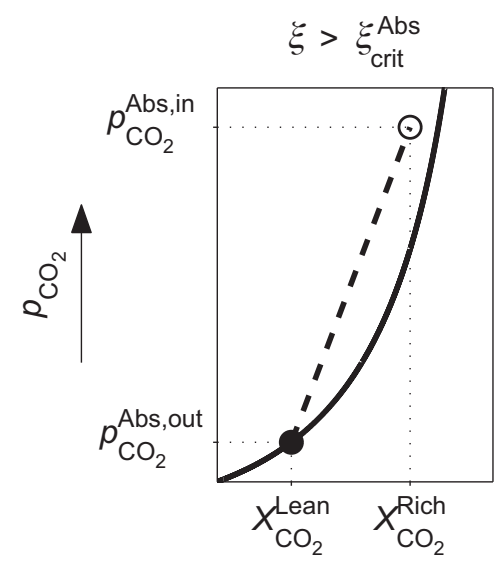

(c)

Figure 3: The three cases that lead to an infinite number of stages in the absorber. (一) equilibrium curve (EC); (--) operating line $(\mathrm{OL})$; $(\bullet)$ pinch point; $(O)$ end point of OL which is not a pinch point. (a) case A1 (one pinch point at the top end of the OL); (b) case A2 (two pinch points at both ends of the OL); (c) case A3 (one pinch point at the bottom end of the OL).

If $\tilde{\xi}_{\min }<\tilde{\xi}<\tilde{\xi}_{\text {crit }}^{\text {Abs }}$ case A1 applies (cf. Figure 3a) and Eq. (11) - Eq. (12) are used for the calculation of $X_{\mathrm{CO}_{2}}^{\mathrm{Lean}}$ and $X_{\mathrm{CO}_{2}}^{\mathrm{Rich}}$.

$$
\begin{aligned}
& X_{\mathrm{CO}_{2}}^{\mathrm{Rich}}=X_{\mathrm{CO}_{2}}^{\mathrm{Abs}, \mathrm{eq}}\left(p_{\mathrm{CO}_{2}}^{\mathrm{Abs}, \mathrm{in}}\right) \\
& X_{\mathrm{CO}_{2}}^{\mathrm{Lean}}=X_{\mathrm{CO}_{2}}^{\mathrm{Rich}}-1 / \tilde{\xi}
\end{aligned}
$$

If $\tilde{\xi}>\tilde{\xi}_{\text {crit }}^{\text {Abs }}$ case A3 applies (cf. Figure 3c) and Eq. (13) - Eq. (14) are used for the calculation of $X_{\mathrm{CO}_{2}}^{\mathrm{Lean}}$ and $X_{\mathrm{CO}_{2}}^{\mathrm{Rich}}$.

$$
\begin{aligned}
& X_{\mathrm{CO}_{2}}^{\mathrm{Lean}}=X_{\mathrm{CO}_{2}}^{\mathrm{Abs} \text { eq }}\left(p_{\mathrm{CO}_{2}}^{\mathrm{Abs} \text { out }}\right) \\
& X_{\mathrm{CO}_{2}}^{\mathrm{Rich}}=X_{\mathrm{CO}_{2}}^{\mathrm{Leen}}+1 / \tilde{\xi}
\end{aligned}
$$

If $\tilde{\xi}=\tilde{\xi}$ crit case A2 applies (cf. Figure 3 b), which is a limiting case of both cases A1 and A3. In this case, either Eq. (11) - Eq. (12) or Eq. (13) - Eq. (14) can be used. The equations Eq. (11) and Eq. (13) represent conditions for an infinite number of stages.

A graphical solution of the absorber step based on McCabe-Thiele plots (cf. Figure 3) is 
possible. The slope of the operating line of the absorber $\theta^{\mathrm{Abs}}$ is proportional to $\tilde{\xi}$ according to Eq. (15).

$$
\theta^{\mathrm{Abs}}=p_{\mathrm{CO}_{2}}^{\mathrm{Abs}, \mathrm{in}} \cdot \psi \cdot \tilde{\xi}
$$

Having calculated the loading of the lean solvent $\left(X_{\mathrm{CO}_{2}}^{\mathrm{Lean}}\right)$ and the loading of the rich solvent $\left(X_{\mathrm{CO}_{2}}^{\mathrm{Rich}}\right)$ one can proceed with the desorber step.

\subsection{Desorber step}

To meet the requirements of the three premises of NoVa, two geometric conditions must be fulfilled in the McCabe-Thiele plot for the desorber: (1) the operating line and the equilibrium curve must have one pinch point; (2) all the points of the operating line must lie below the equilibrium curve. Futhermore the condition (3), that the reboiler is an equilibrium stage, has to be considered.

This results in the three cases shown in Figure 4. In principle, it is also possible that a pinch occurs at the lower end of the operating line (i.e. the pinch in the reboiler). It can be shown that this special case occurs for $X_{\mathrm{CO}_{2}}^{\mathrm{Lean}}=0$, i.e. $\psi=1$. This special case is not considered here.

The task is now to find the slope of the desorber operating line $\theta^{\text {Des }}$ for each of the three cases shown in Figure 4, $\theta^{\text {Des }}$ characterizes the internal flowrates of gas and liquid in the desorber and is required in the subsequent step. $\theta^{\text {Des }}$ is calculated with Eq. (16) where $p_{\mathrm{CO}_{2}}^{\text {Des,pinch }}$ and $X_{\mathrm{CO}_{2}}^{\text {Des,pinch }}$ are the partial pressure of $\mathrm{CO}_{2}$ and the $\mathrm{CO}_{2}$ loading in the desorber corresponding to the pinch point, which is determined differently depending on the case. The equilibrium condition for the pinch point is given by Eq. (17)

$$
\begin{gathered}
\theta^{\text {Des }}=\frac{p_{\mathrm{CO}_{2}}^{\text {Des,pinch }}}{X_{\mathrm{CO}_{2}}^{\text {Des,pinch }}-X_{\mathrm{CO}_{2}}^{\mathrm{Lean}}} \\
p_{\mathrm{CO}_{2}}^{\text {Des,pinch }}=p_{\mathrm{CO}_{2}}^{\text {Des,eq }}\left(X_{\mathrm{CO}_{2}}^{\text {Des,pinch }}\right)
\end{gathered}
$$




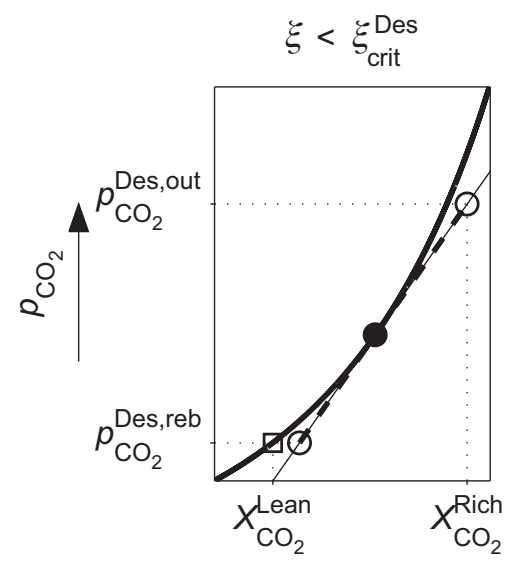

(a)

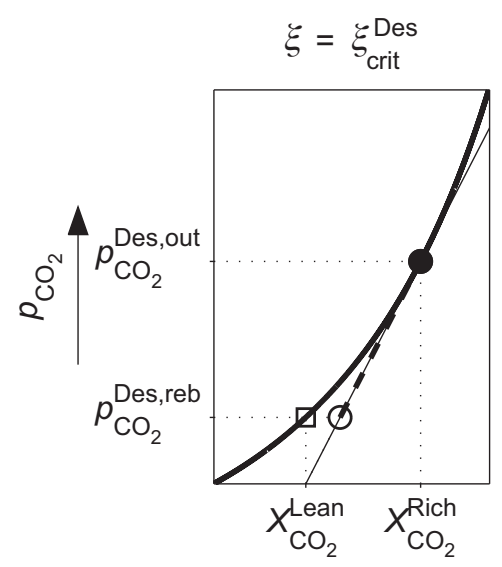

(b)

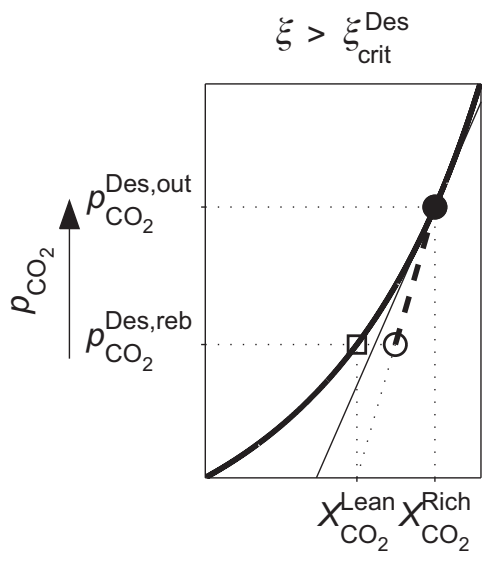

(c)

Figure 4: The three cases that lead to an infinite number of stages in the desorber. (-) equilibrium curve (EC); (--) operating line (OL); thin (-) tangent to EC in the pinch point; ( $\bullet$ ) pinch point; (O) end point of OL which is not a pinch point; ( $\square$ ) reboiler state. (a) Case D1 (pinch point on the OL, OL tangent to EC); (b) case D2 (pinch point at the top end of the OL, OL tangent to EC; (c) case D3 (pinch point at the top end of the OL, OL not tangent to EC).

In case D1 (cf. Figure 4a), the operating line is a tangent to the equilibrium line in the pinch point that is not an endpoint of the operating line. In case D2 (cf. Figure 4b), the pinch point is the top end of the operating line and the operating line is a tangent to the equilibrium line. In case D3 (cf. Figure 4c), the pinch point is the top end of the operating line and the operating line is not a tangent to the equilibrium line.

Considering the reboiler of the absorber as one theoretical stage, the partial pressure of $\mathrm{CO}_{2}$ in the reboiler $\left(p_{\mathrm{CO}_{2}}^{\text {Des,reb}}\right)$ is the partial pressure of $\mathrm{CO}_{2}$ in equilibrium with the lean solvent (cf. Eq. (18)).

$$
p_{\mathrm{CO}_{2}}^{\text {Des,reb }}=p_{\mathrm{CO}_{2}}^{\text {Des,eq }}\left(X_{\mathrm{CO}_{2}}^{\mathrm{Lean}}\right)
$$

Which of the three cases described above applies, depends on the value $\tilde{\xi}$ crit as defined by Eq. (19). $p_{\mathrm{CO}_{2}}^{\text {Des,max }}$ is the maximum partial pressure of $\mathrm{CO}_{2}$ that can be reached in the desorber, which is the equilibrium partial pressure of $\mathrm{CO}_{2}$ corresponding to the rich loading at the desorber 
temperature (cf. Eq. (20)).

$$
\begin{gathered}
\xi_{\text {crit }}^{\text {Des }}=\left.\frac{1}{p_{\mathrm{CO}_{2}}^{\text {Des,max }}} \cdot \frac{\mathrm{d} p_{\mathrm{CO}_{2}}^{\text {Des,eq }}\left(X_{\mathrm{CO}_{2}}\right)}{\mathrm{d} X_{\mathrm{CO}_{2}}}\right|_{X_{\mathrm{CO}_{2}}=X_{\mathrm{CO}_{2}}^{\mathrm{Rich}}} \\
p_{\mathrm{CO}_{2}}^{\text {Des,max }}=p_{\mathrm{CO}_{2}}^{\text {Des,eq }}\left(X_{\mathrm{CO}_{2}}^{\mathrm{Rich}}\right)
\end{gathered}
$$

If $\tilde{\xi}<\tilde{\xi}_{\text {crit }}^{\text {Des }}$ case D1 applies (cf. Figure 4a). The desorber is then described by a nonlinear system of two equations (cf. Eq. (16) and Eq. (21)), with two unknowns: $\theta^{\text {Des }}$ and $p_{\mathrm{CO}_{2}}^{\text {Des,pinch }}$, which have to be solved simultaneously.

$$
\theta^{\text {Des }}=\left.\frac{\mathrm{d} p_{\mathrm{CO}_{2}}^{\text {Des,eq }}\left(X_{\mathrm{CO}_{2}}\right)}{\mathrm{d} X_{\mathrm{CO}_{2}}}\right|_{X_{\mathrm{CO}_{2}}=X_{\mathrm{CO}}^{\text {Des,pinch }}}
$$

If $\tilde{\xi}>\tilde{\xi}_{\text {crit }}^{\text {Des }}$ case D3 applies (cf. Figure 4c), $X_{\mathrm{CO}_{2}}^{\text {Des, pinch }}=X_{\mathrm{CO}_{2}}^{\mathrm{Rich}}$ and $\theta^{\text {Des }}$ is calculated with Eq. (22).

$$
\theta^{\text {Des }}=\frac{p_{\mathrm{CO}_{2}}^{\text {Des,max }}}{X_{\mathrm{CO}_{2}}^{\text {Rich }}-X_{\mathrm{CO}_{2}}^{\text {Lean }}}
$$

If $\tilde{\xi}=\tilde{\xi}$ crit case D2 applies (cf. Figure 4b). It is a limiting case of both cases D1 and D3. Hence, $\theta^{\text {Des }}$ can be calculated either with Eq. (16) and Eq. (21) or from Eq. (22). The auxiliary lines in Figure 4 suggest a straightforward graphical solution of the desorber step.

Having calculated $p_{\mathrm{CO}_{2}}^{\text {Des,reb }}$ and $\theta^{\text {Des }}$ one can now proceed with the energy balance step.

\subsection{Energy balance step}

In this step, the specific regeneration energy $q^{\text {Reb }}$ is calculated from the energy balance of the desorber according to Eq. (23), which is based on Notz et al. $\underline{1}$ There are three contributions: the enthalpy of $\mathrm{CO}_{2}$ desorption $(\mathrm{R})$, the energy for heating the solvent $(\mathrm{S})$, and the energy for heating and evaporating the water reflux $(\mathrm{W})$. The coefficients $C_{\mathrm{R}}, C_{\mathrm{S}}$ and $C_{\mathrm{W}}$ depend on caloric properties 
and operating parameters (Eqs. (24) to (26)) as shown by Notz et al. 1

$$
\begin{gathered}
q^{\mathrm{Reb}}=C_{\mathrm{R}}+C_{\mathrm{S}} \cdot \tilde{\xi}+C_{\mathrm{W}} \cdot \tilde{\xi} \cdot \frac{p^{\text {Des }}-p_{\mathrm{CO}}^{\text {Des,reb }}}{\theta^{\text {Des }}} \\
C_{\mathrm{R}}=-\frac{\Delta_{\mathrm{abs}} h_{\mathrm{CO}_{2}}^{\mathrm{S}}}{M_{\mathrm{CO}_{2}}} \\
C_{\mathrm{S}}=\frac{c_{\mathrm{p}, \mathrm{S}}^{\mathrm{L}} \cdot\left(T^{\text {Des,lean }}-T^{\text {Des,rich }}\right)}{M_{\mathrm{CO}_{2}}} \\
C_{\mathrm{W}}=\frac{\Delta_{\text {vap }} h_{\mathrm{H}_{2} \mathrm{O}}+M_{\mathrm{H}_{2} \mathrm{O}} \cdot c_{\mathrm{p}, \mathrm{H}_{2} \mathrm{O}}^{\mathrm{L}} \cdot\left(T^{\text {Des,rich }}-T^{\text {Cond }}\right)}{M_{\mathrm{CO}_{2}}}
\end{gathered}
$$

$c_{\mathrm{p}, \mathrm{H}_{2} \mathrm{O}}^{\mathrm{L}}$ and $c_{\mathrm{p}, \mathrm{S}}^{\mathrm{L}}$ are the specific heat capacities of liquid water and of the liquid solvent, respec-

tively. $\Delta_{\text {abs }} h_{\mathrm{CO}_{2}}^{\mathrm{S}}$ is the molar enthalpy of absorption of $\mathrm{CO}_{2}$ in the solvent $\mathrm{S}$ and $\Delta_{\mathrm{vap}} h_{\mathrm{H}_{2} \mathrm{O}}$ is the molar enthalpy of vaporization of water. $M_{\mathrm{H}_{2} \mathrm{O}}$ and $M_{\mathrm{CO}_{2}}$ are the molar masses of water and carbon dioxide, respectively. For the temperatures $T^{\text {Des,lean }}, T^{\text {Des,rich }}$ and $T^{\text {Cond }}$, see Figure 1 ,

\section{SolSOFT correlation of gas solubility data}

SolSOFT is an empirical equation that was developed in the present work for describing gas solubilities in reactive systems. The SolSOFT equation (Eq. (27) describes the loading of the solvent with the gas $\mathrm{G} \alpha_{\mathrm{G}}$ as a function of the partial pressure of the gas $p_{\mathrm{G}}$ at a fixed temperature. It has four positive parameters $\left(K_{\mathrm{p}}\right.$ and $K_{\mathrm{c}}$, which both have the unit of a pressure, and $n$ and $m$, which are dimensionless).

$$
\alpha_{\mathrm{G}}=\left(p_{\mathrm{G}} / K_{\mathrm{p}}\right)^{m}+n \cdot \frac{\left(p_{\mathrm{G}} / K_{\mathrm{c}}\right)^{n}}{1+\left(p_{\mathrm{G}} / K_{\mathrm{c}}\right)^{n}}
$$

The most relevant intrinsic properties of SolSOFT are: it is differentiable, it passes through the origin, and is monotonously increasing. Because of its monotonicity the function $\alpha_{\mathrm{G}}\left(p_{\mathrm{G}}\right)$ can 
be inverted such that also $p_{\mathrm{G}}\left(\alpha_{\mathrm{G}}\right)$ is known. The analytical function $\alpha_{\mathrm{G}}\left(p_{\mathrm{G}}\right)$ is explicit, whereas the inverse function $p_{\mathrm{G}}\left(\alpha_{\mathrm{G}}\right)$ is only implicitly defined by Eq. (27). Nevertheless, $p_{\mathrm{G}}\left(\alpha_{\mathrm{G}}\right)$ is welldefined by Eq. (27) and can be easily determined numerically. Moreover, because of the monotonicity of $\alpha_{\mathrm{G}}\left(p_{\mathrm{G}}\right)$ also $p_{\mathrm{G}}\left(\alpha_{\mathrm{G}}\right)$ is monotonous, and, as $\alpha_{\mathrm{G}}\left(p_{\mathrm{G}}\right)$ is generally concave, $p_{\mathrm{G}}\left(\alpha_{\mathrm{G}}\right)$ is generally convex.

For a detailed discussion of the convexity of SolSOFT see Supplementary Information B. $X_{\mathrm{G}}\left(p_{\mathrm{G}}\right)$ can be determined from $\alpha_{\mathrm{G}}\left(p_{\mathrm{G}}\right)$ using Eq. (6).

The first term in the SolSOFT Eq. (27) can be interpreted as a physical contribution and the second term as a chemical contribution. The parameter $n$ in the second term is related to the stoechiometric coefficient in the reaction of $n$ molecules of the gas $\mathrm{G}$ with a solvent molecule to form a $n: 1$ complex. At low loadings the chemical term usually dominates. The physico-chemical background of SolSOFT and a variant of Eq. (27) with only a chemical term are discussed in the Appendix A.

A drawback of the SolSOFT Eq. (27) is that its behavior regarding the tangent of $p_{\mathrm{G}}\left(\alpha_{\mathrm{G}}\right)$ at low pressures is only well-behaved for $m=1$. However, in practical applications good fits of experimental data are achieved also when $m$ is used as adjustable parameter, as shown below. A version of the SolSOFT equation that circumvents this drawback is described in the Appendix A. All calculations of the present work were carried out with the simple form of SolSOFT presented in Eq. (27).

It is sometimes convenient to use temperature-dependent parameters in SolSOFT. The following has turned out to be useful: a temperature dependence of the van't Hoff type is used for $K_{\mathrm{p}}$ and $K_{\mathrm{c}}$ (cf. Eq. (28) and Eq. (29)), while $m$ and $n$ are constant.

$$
\begin{aligned}
& \ln \left(K_{\mathrm{p}} / \text { bar }\right)=a_{\mathrm{p}}+\frac{b_{\mathrm{p}}}{(T / \mathrm{K})} \\
& \ln \left(K_{\mathrm{c}} / \text { bar }\right)=a_{\mathrm{c}}+\frac{b_{\mathrm{c}}}{(T / \mathrm{K})}
\end{aligned}
$$


The differential enthalpy of absorption of the gas $\mathrm{G}$ into the solvent $\mathrm{S}$ can be calculated based on gas solubility data using a Gibbs-Helmholtz equation 12 (Eq. (30)),

$$
\Delta_{\mathrm{abs}}^{\mathrm{diff}} h_{\mathrm{G}}^{\mathrm{S}}=\mathrm{R} \cdot\left(\frac{\partial \ln \left(p_{\mathrm{G}}\right)}{\partial(1 / T)}\right)_{\alpha_{\mathrm{G}}}
$$

where $\mathrm{R}$ is the universal gas constant.

Based on SolSOFT with temperature-dependent parameters, explicit algebraic expressions for the calculation of both the differential and integral enthalpy of absorption of $G$ into $S$ were derived in Supplementary Information C and are presented in the Appendix B.

\section{Results and discussion}

To illustrate the results that can be obtained with NoVa, the method was applied in the present work for ranking MEA30, MDEA30 and BuTAD30 as solvents for $\mathrm{CO}_{2}$ absorption from flue gas. MEA30, MDEA30 and BuTAD30 stand for aqueous solutions of monoethanolamine, methyldiethanolamine and butyltriacetonediamine, respectively, with $x_{\mathrm{Amine}}^{(\mathrm{m}), \mathrm{S}^{*}}=0.3 \mathrm{~g} \cdot \mathrm{g}^{-1}$. The mass fractions of amine refer to the unloaded solvent $\mathrm{S}^{*}$. We emphasize that the main purpose of presenting the results is not the actual ranking of the three solvents but rather to give an example for how the new short-cut method NoVa can be applied and which type of results can be obtained with this new computational method.

Following Notz et al., $\frac{1}{2}$ the caloric data used in the present work are: heat capacity of liquid water $c_{\mathrm{p}, \mathrm{H} 2 \mathrm{O}}^{\mathrm{L}}=4.2 \mathrm{~kJ} \cdot \mathrm{kg}^{-1} \mathrm{~K}^{-1}$, enthalpy of vaporization of water $\Delta_{\mathrm{vap}} h_{\mathrm{H}_{2} \mathrm{O}}=39.8 \mathrm{~kJ} \cdot \mathrm{mol}^{-1}$, and heat capacity of all solvents $c_{\mathrm{p}, \mathrm{S}}^{\mathrm{L}}=4 \mathrm{~kJ} \cdot \mathrm{kg}^{-1} \mathrm{~K}^{-1}$. The remaining solvent-related input of NoVa, namely the two $\mathrm{CO}_{2}$ solubility isotherms and the enthalpy of absorption of $\mathrm{CO}_{2}$ are calculated with the SolSOFT equation. Two versions were studied: one with an individual parameter set for each isotherm, and one using the temperature-dependent correlations of the parameters. The gas solubility data to which the SolSOFT equation was fitted was taken from literature. The experimental data of Wagner et al. $\underline{13}$ was used for MEA30. The model of Ermatchkov et al., 14 which 
is based on the extended Pitzer equations, was used for calculating data points for MDEA30. The experimental data of Vasiliu et al. $\stackrel{10}{\underline{1}}$ was used for BuTAD30.

\subsection{Application of the SolSOFT equation}

Figure 5 shows experimental data for the solubility of $\mathrm{CO}_{2}$ in MEA30 at $393 \mathrm{~K}$ from Wagner et al. 13 together with results from their correlation with the SolSOFT equation. For comparison, also data from two other empirical correlations (polynomial and sigmoid, see Supplementary Information D) and from a physico-chemical model based on the extended Pitzer equation 13 are shown. Also the polynomial and the sigmoid correlations were fitted to the data shown in Figure 5, while the phyisco-chemical model ${ }^{13}$ was fitted to a larger data set. In the range where experimental data is available, all models show a similar performance. But outside that range, the model results differ strongly. From the four tested models, SolSOFT is the only one which yields an expected monotonically increasing gas solubility in the entire range, which is shown in Figure 5 .

The SolSOFT equation with temperature-dependent parameters can be used for calculating the enthalpy of absorption, cf. Eq. (42) from Appendix B. An example is presented in Supplementary Information $\mathrm{C}$, which shows the differential enthalpy of absorption of $\mathrm{CO}_{2}$ in $\mathrm{MEA} 30$ at $393 \mathrm{~K}$ as a function of the $\mathrm{CO}_{2}$ loading. The SolSOFT results are based on a fit to the experimental data of Wagner et al. $\underline{13}$ at temperatures between $313 \mathrm{~K}$ and $393 \mathrm{~K}$. For comparison, the figure in Supplementary Information $\mathrm{C}$ shows also the experimental data determined by Kim and Svendsen 15 and the predictions of von Harbou 16 based on the physico-chemical model of Wagner et al. 13 The results obtained from SolSOFT are satisfactory, considering the simplicity of the procedure.

$\mathrm{CO}_{2}$ gas solubility isotherms for the solvents MEA30, MDEA30 and BuTAD30 were fitted with the SolSOFT equation with constant as well as with temperature-dependent parameters. The results are presented in Supplementary Information E. The results obtained with the temperaturedependent form are compared to the experimental data to which they were fitted in Figure 6, The agreement is good.

From the temperature-dependent SolSOFT correlation integral enthalpies of absorption of $\mathrm{CO}_{2}$ 


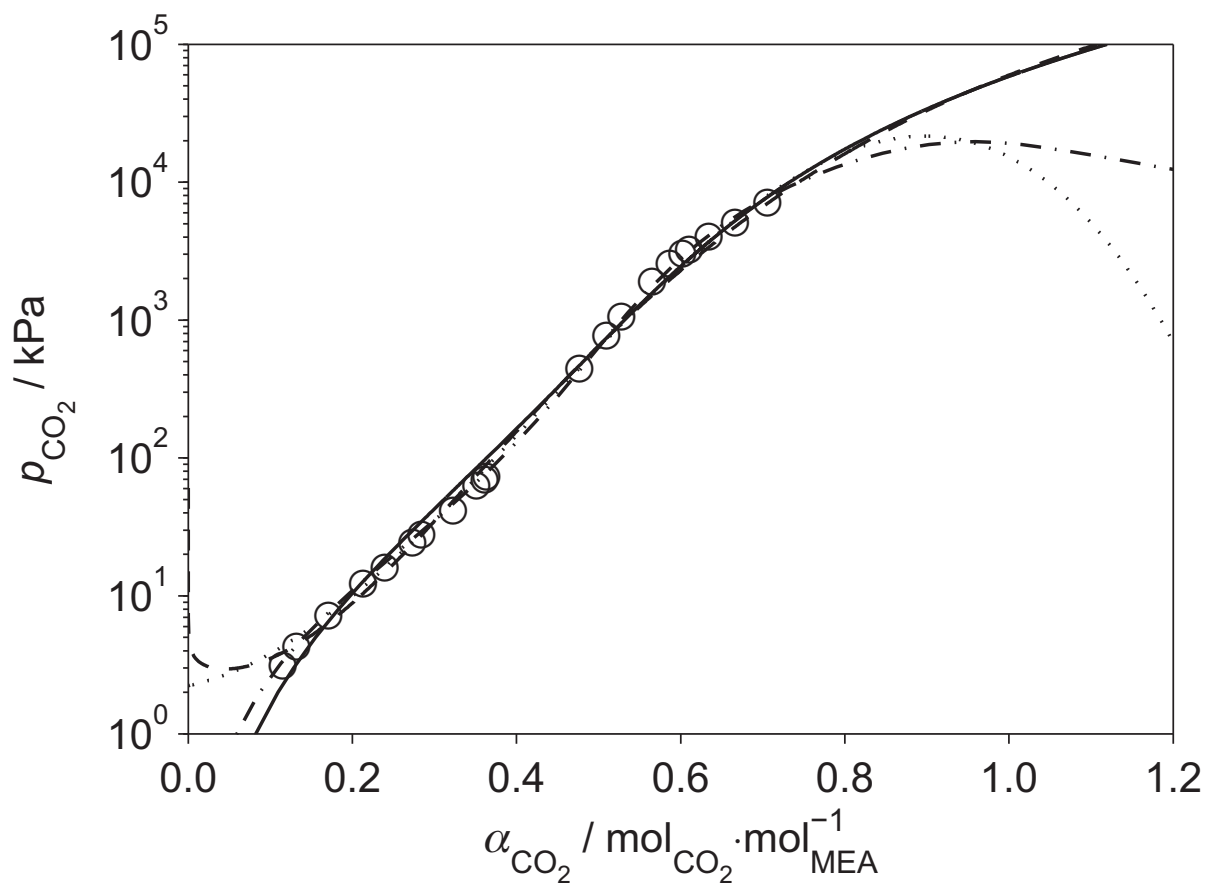

Figure 5: Equilibrium data for the solubility of $\mathrm{CO}_{2}$ in MEA30 at $393 \mathrm{~K}$ : partial pressure of $\mathrm{CO}_{2}$ as a function of the $\mathrm{CO}_{2}$ loading of the solvent. (O) experimental data. $\underline{13}$ Correlations of the present data: (-) SolSOFT correlation; (- ) sigmoid; (..) polynomial. $(\cdot-)$ physico-chemical model. $\underline{13}$ 


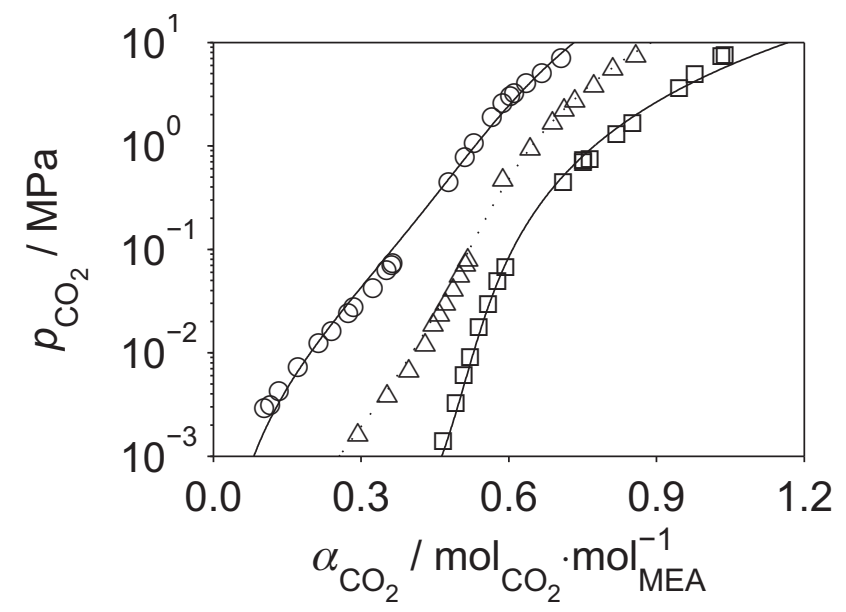

(a)

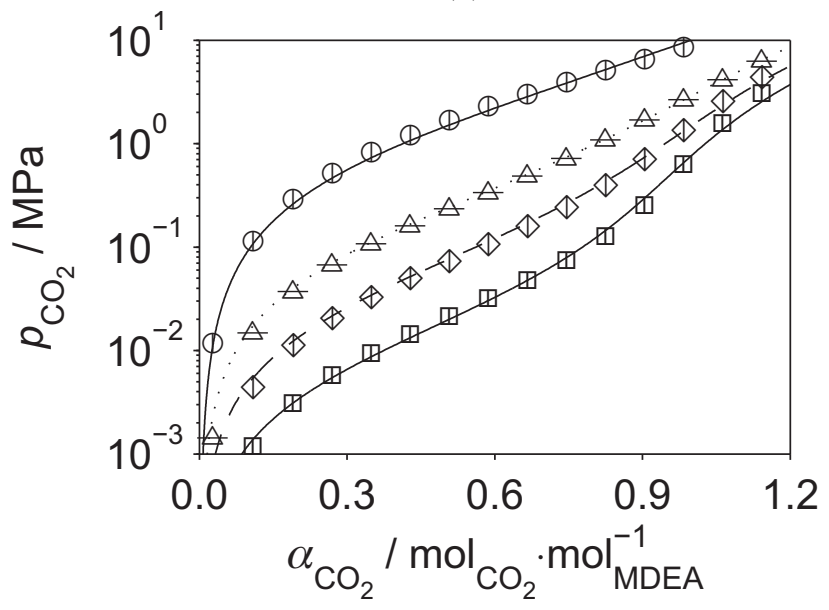

(b)

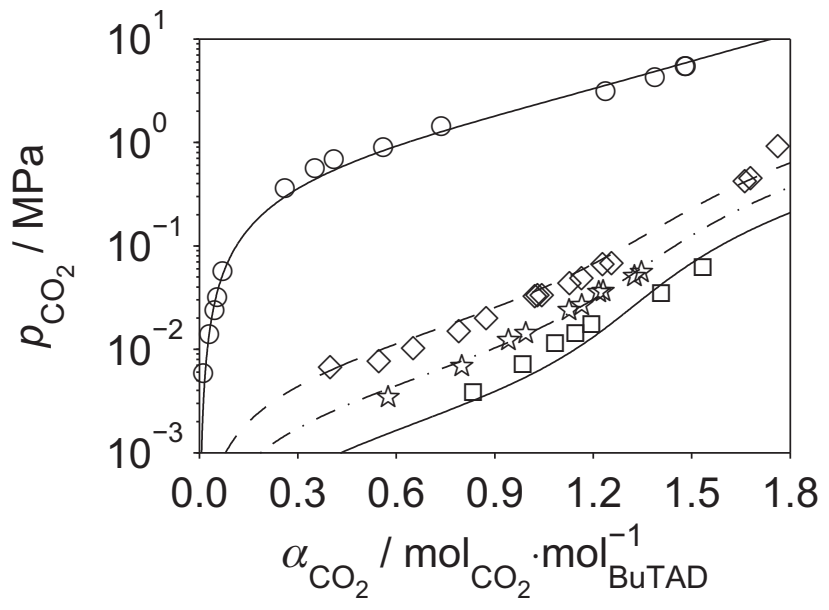

(c)

Figure 6: Equilibrium data for the solubility of $\mathrm{CO}_{2}$ in different amines: partial pressure of $\mathrm{CO}_{2}$ as a function of the $\mathrm{CO}_{2}$ loading of the solvent. Lines: SolSOFT correlation with temperaturedependent parameters. Symbols: literature data. Top: MEA30 (313, 353, 393) K, experimental data from Wagner et al. 13 Middle: MDEA30 $(313,333,353,393) \mathrm{K}$, data from a physico-chemical model. 14 Bottom: BuTAD30 $(313,323,333,393) \mathrm{K}$, experimental data from Vasiliu et al. 10 
in the three solvents were determined. The integral is evaluated for the interval of the $\mathrm{CO}_{2}$ partial pressure from 0 to 2 bar using Eq. (46) from Appendix B. The results are: $\Delta_{\mathrm{abs}} h_{\mathrm{CO}_{2}}^{\mathrm{MEA} 30}=$ $-88 \mathrm{~kJ} \cdot \mathrm{mol}_{\mathrm{CO}_{2}}^{-1}, \Delta_{\mathrm{abs}} h_{\mathrm{CO}_{2}}^{\mathrm{MDEA30}}=-45 \mathrm{~kJ} \cdot \mathrm{mol}_{\mathrm{CO}_{2}}^{-1}, \Delta_{\mathrm{abs}} h_{\mathrm{CO}_{2}}^{\mathrm{BuTAD} 30}=-67 \mathrm{~kJ} \cdot \mathrm{mol}_{\mathrm{CO}_{2}}^{-1} \cdot$ These numbers are used in the following in the NoVa method.

The SolSOFT equation was also used for correlating experimental data for the solubility of $\mathrm{CO}_{2}$ in many other aqueous amine systems, which were taken from the literature. The results are reported in the Supplementary Information E.

\subsection{Studies with the NoVa method}

The NoVa method and the method of Notz et al. ${ }^{\underline{1}}$ were tested using MEA30 as a solvent in two scenarios BC1 and BC2 which are taken from Mangalapally et al. $\stackrel{17}{\underline{T}}$ The flue gas of scenario BC1 corresponds to a gas-fired power plant and that of scenario BC2 to a coal-fired power plant. 17 Table 1 gives details. For the calculations with the method of Notz et al. $\underline{1}$ the number of stages were taken as used in their publication: 15 for the absorber and 10 for the desorber. For calculating the gas solubility with the NoVa method, the individual SolSOFT fits at $313 \mathrm{~K}$ and $393 \mathrm{~K}$ were used. Figure 7 shows the results of the NoVa method and those of Notz et al. $\underline{1}$ together with the corresponding experimental data of Mangalapally et al. $\underline{17}$ in a plot of the regeneration energy $q^{\text {Reb }}$ as a function of $L / G$ for the cases $\mathrm{BC} 1$ and $\mathrm{BC} 2$. The results of both methods are in close agreement for $\mathrm{BC} 1$ as well as for $\mathrm{BC} 2$. Furthermore, the predictions of both methods match the experimental data of Mangalapally et al. $\stackrel{17}{ }$ reasonably, given the simplicity and strong assumptions of the NoVa method. Results from a simple method like NoVa cannot be expected to agree quantitatively with experimental plant data. It is more important, that they predict trends reasonably well. This is true for the cases studied in Figure 7. The good agreement between the results of the method of Notz et al. $\stackrel{1}{ }$ and those of the new NoVa method for the cases studied in Figure 7 should not lead to the conclusion that there is no difference between the two methods. The method of Notz et al. 1 contains simulation parameters that need to be set empirically and its results also depend on the way the solubility isotherms are discretized. All this is not required in NoVa. Furthermore, 


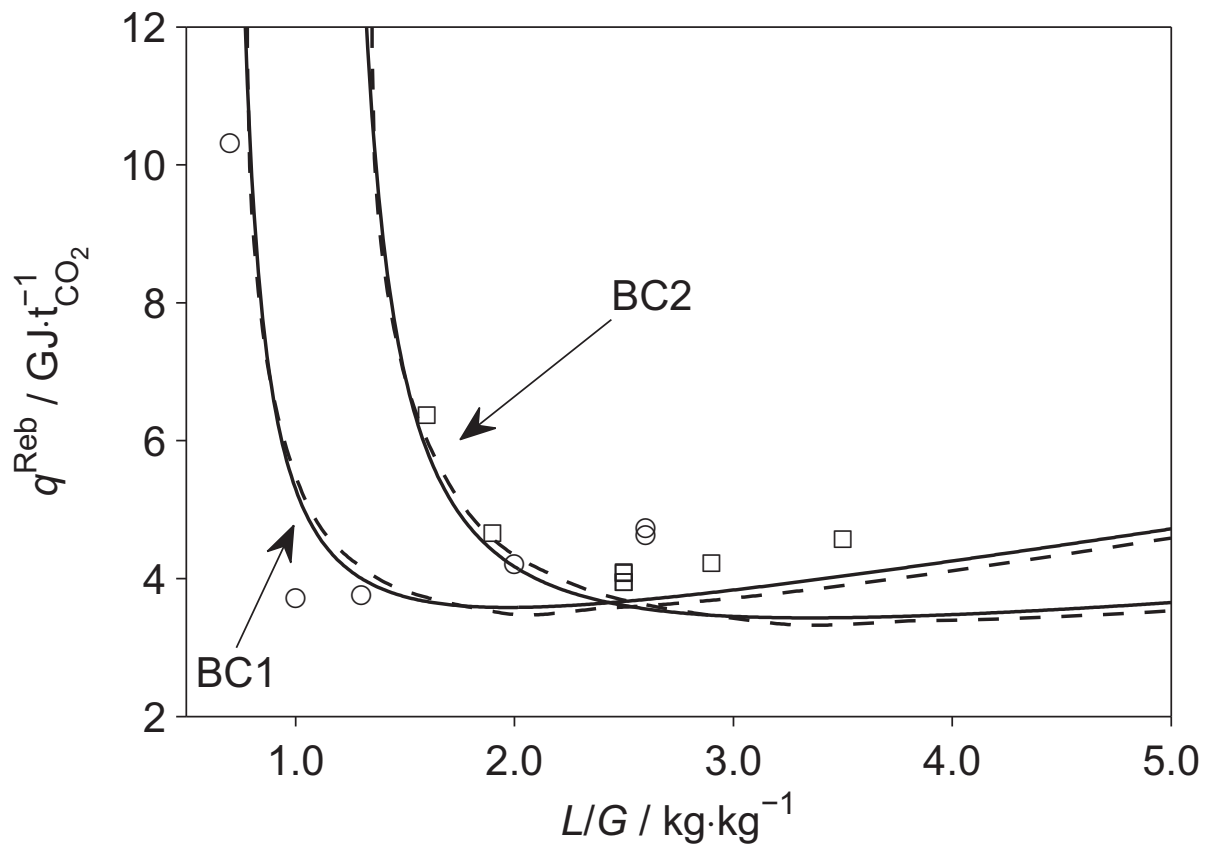

Figure 7: Specific regeneration energy as a function of $L / G$ for the solvent MEA30 in the scenarios BC1 and BC2 and $\psi=0.9$. Predictions: (-) NoVa method, (--) method of Notz et al. $\frac{1}{r}$ Results from pilot plant experiments: $\underline{18}$ (O) $\mathrm{BC} 1,(\square) \mathrm{BC} 2 . \underline{18}$

the simple equations of NoVa are much easier to implement than those of the method of Notz et al. $\underline{1}$ and the numerical solution of the NoVa equations is faster and distinctly more robust. Last not least, as demonstrated below, the NoVa method gives structural insight into the absorption / desorption process that could not be obtained with the method of Notz et al. $\frac{1}{}$ Some information on the in silico implementation of NoVa method is given in Appendix C.

The assumption of isothermality of the desorber can lead to unphysical values of the partial pressure of $\mathrm{CO}_{2}$, which are higher than the total pressure for both the method of Notz et al. $\underline{\underline{1}}$ and NoVa. This was already discussed by Notz et al., $\underline{\underline{1}}$ who concluded that it barely affects the solvent ranking.

Plots of the type $q^{\mathrm{Reb}}(L / G)$ can be calculated for any solvent and scenario for a given removal rate $\psi$. The NoVa method yields insight in the structure of these plots. Under the assumptions of 
Table 1: Scenarios BC1 (gas fired power plant) and BC2 (coal fired power plant). 17

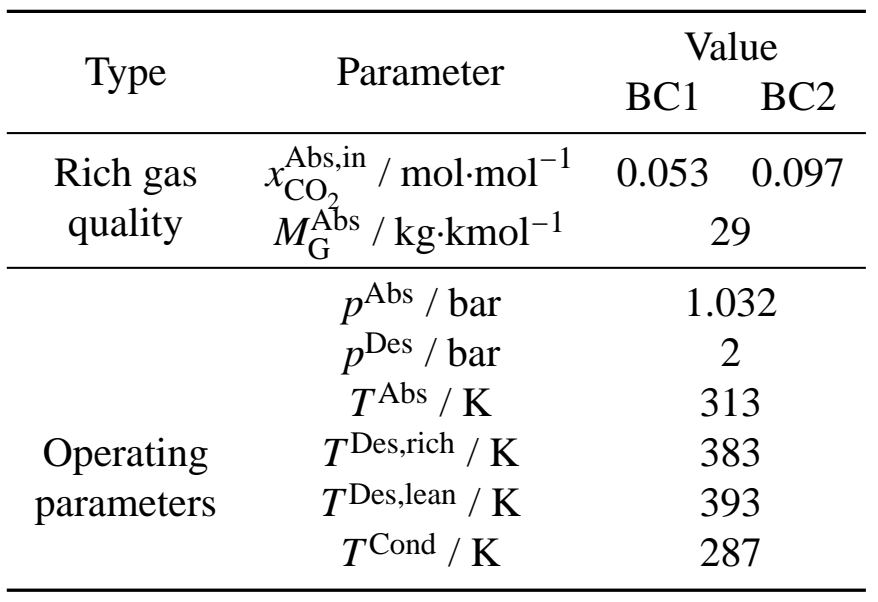

the NoVa method, namely infinite number of stages in both the absorber and desorber, the curve $q^{\operatorname{Reb}}(L / G)$ may have different branches. These branches result from the fact that with changing $L / G$, different cases for the absorber and desorber pinch may apply, as discussed above. Some of these branches can be discerned easily in the plots of $q^{\operatorname{Reb}}(L / G)$ as they join sharply in nondifferentiable points. This is true for case changes in the absorber. The case changes in the desorber are usually not discernible optically as the corresponding branches join smoothly in differentiable points. The first-order differentiability of $q^{\mathrm{Reb}}$ in the desorber case changes points is discussed in Supplementary Information F. The NoVa method yields not only information on the reboiler duty $q^{\mathrm{Reb}}$, that enables drawing plots like those shown in Figure 7 also the individual contributions to the reboiler energy are available. An example is given in the Supplementary Information G. Furthermore, NoVa also yields information on all streams in the flow sheet, e.g., numbers for the rich and lean loading. Also the cyclic capacity can be determined easily. An example is given in the Supplementary Information H.

Instead of studying $q^{\operatorname{Reb}}(L / G)$, also $q^{\operatorname{Reb}}(\xi)$ can be studied where $\xi=(L / G) / \psi$, as defined in Eq. (4). Figure 8 shows a sketch of the curves $q^{\operatorname{Reb}}(\xi)$ for a given scenario and different values of the removal rate $\psi$. For $\psi \rightarrow 0$ the plot $q^{\operatorname{Reb}}(\xi)$ is $\mathrm{U}$-shaped and has no branches. For any finite value of $\psi$ the function $q^{\operatorname{Reb}}(\xi)$ has two branches: for low number of $\xi$ it coincides with the curve for $\psi \rightarrow 0$. For values above a certain critical value of $\xi$ the function $q^{\operatorname{Reb}}(\xi)$ follows a second 


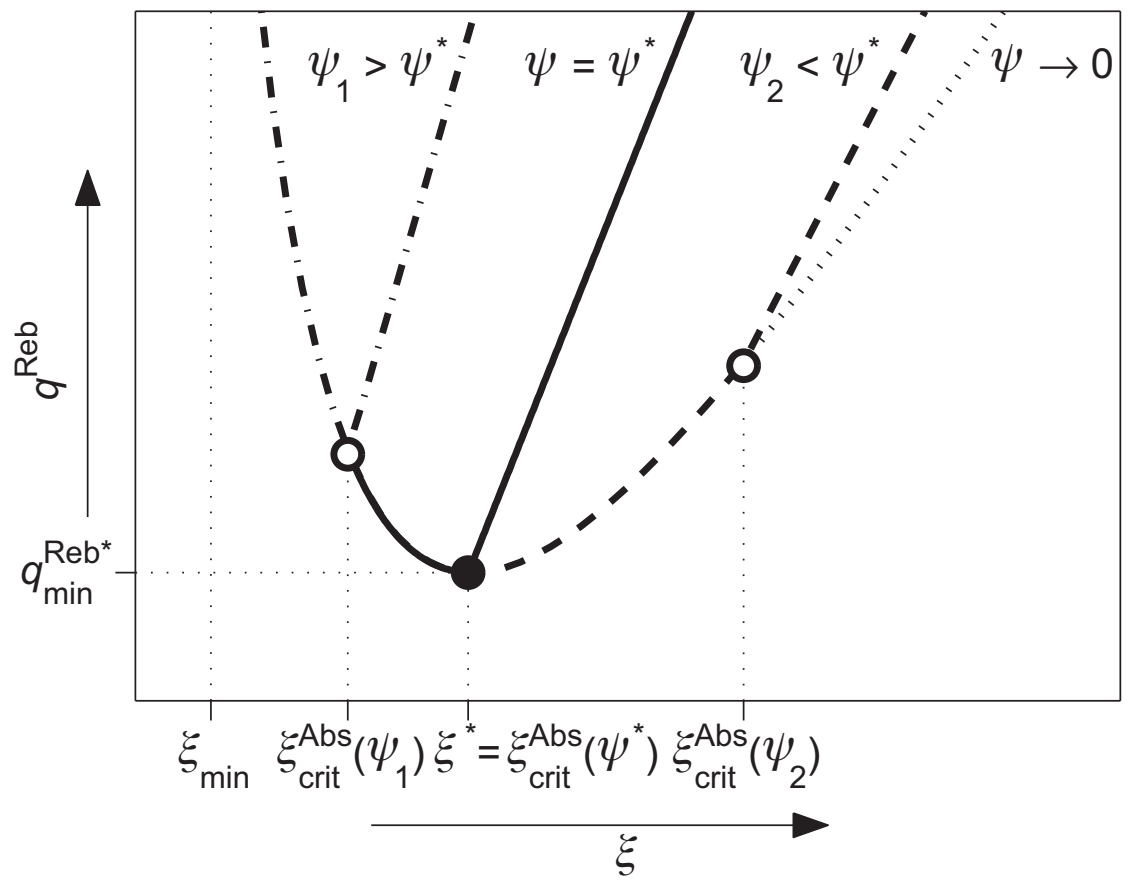

Figure 8: Sketch of the specific regeneration energy $q^{\mathrm{Reb}}$ as a function of $\xi$ (cf. Eq. (4)) for different values of the removal rate $\psi$ obtained with the NoVa method. Each curve $q^{\mathrm{Reb}}(\xi)$ has two branches. One branch is a part of a common U-shaped envelope, which is the curve $q^{\operatorname{Reb}}(\xi)$ for $\psi \rightarrow 0$. The second branch is almost straight and depends on the number of $\psi$. The intersection of the two branches is called sharp point here. Three sharp points are marked in the diagram. There is a global optimum for which the specific regeneration energy is minimum (๑). It is found for $\psi=\psi^{*}$ and $\xi=\xi^{*}$. The point $(\bullet)$ is also the optimal point for all curves $q^{\operatorname{Reb}}(\xi)$ for $\psi<\psi^{*}$, while for $\psi>\psi^{*}$ the optimum is the corresponding sharp point. There is a minimum value of $\xi$ below which no solutions exist. 
branch, that depends on the value of $\psi$. The critical value is given by $\xi_{\text {crit }}^{\text {Abs }}$ and can be calculated from Eq. (9). From Figure 8 it is clear that for all values of $\psi$ the function $q^{\operatorname{Reb}}(\xi)$ is U-shaped and that for each $\psi$ there is a certain minimum value $q_{\mathrm{min}}^{\mathrm{Reb}}$, as it is also well known from the plots of $q^{\operatorname{Reb}}(L / G)$. However, Figure 8 contains substantial new information. The curves $q^{\operatorname{Reb}}(\xi)$ for the different numbers $\psi$ have a common envelope and basically differ only in the position of their second branch. There is a global optimum $q_{\mathrm{min}}^{\mathrm{Reb} *}$ with a corresponding number $\xi *$ which found for a certain $\psi^{\star}$ (full point in Figure 8). The value $q_{\min }^{\mathrm{Reb}^{*}}$ is the lowest attainable specific regeneration energy for the studied scenario and solvent. It follows from Figure 8 that $q_{\mathrm{min}}^{\mathrm{Reb} *}$ is also the minimum specific regeneration energy for all $\psi<\psi^{*}$. For any $\psi>\psi^{*}$, the minimum reboiler duty $q_{\min }^{\mathrm{Reb}}$ is larger than $q_{\mathrm{min}}^{\mathrm{Reb} *}$. It is found at the intersection of the two branches of the curve $q^{\mathrm{Reb}}(\xi)$ for the given $\psi>\psi^{*}$. Such intersections also exist for $\psi<\psi^{\star}$, but the corresponding specific regeneration energies are not minima on the $q^{\operatorname{Reb}}(\xi)$ curve.

Figure 8 shows that there are different types of solutions of the absorber / desorber model under the assumptions of the NoVa model for a given solute, solvent and scenario. They result from the different cases for the absorber and desorber operation (cf. Figure 3 and Figure 4), which can be combined in different ways. An overview of the topology of the possible solutions can be given as a function of the variables $\psi$ and $\xi$. The resulting topology (cf. Figure 9 ) is discussed in the Supplementary Information I.

\section{$5.3 q_{\text {min }}^{\text {Reb* }}, \psi^{\star}$ and $\xi^{*}$ as new criteria for assessing solvents}

For any solute, solvent and scenario, the numbers for $q_{\min }^{\mathrm{Reb}}, \psi^{*}$ and $\xi^{*}$ can be determined as described above. These numbers are helpful for assessing solvents. Table 2 shows the results obtained for MEA30, MDEA30 and BuTAD30 and the scenario BC2 using the NoVa method together with the SolSOFT correlations presented above. Compared to MEA30, MDEA30 has a much lower $q_{\mathrm{min}}^{\mathrm{Reb}}$ and only a slightly higher $\xi^{*}$. The attractiveness of BuTAD30 is confirmed. It has by far the lowest $\xi^{*}$ and a $q_{\mathrm{min}}^{\mathrm{Reb*}}$ which is slightly lower than that of MDEA30. If the high purity of the lean gas were the main target for a gas purification process, the order of magnitude 


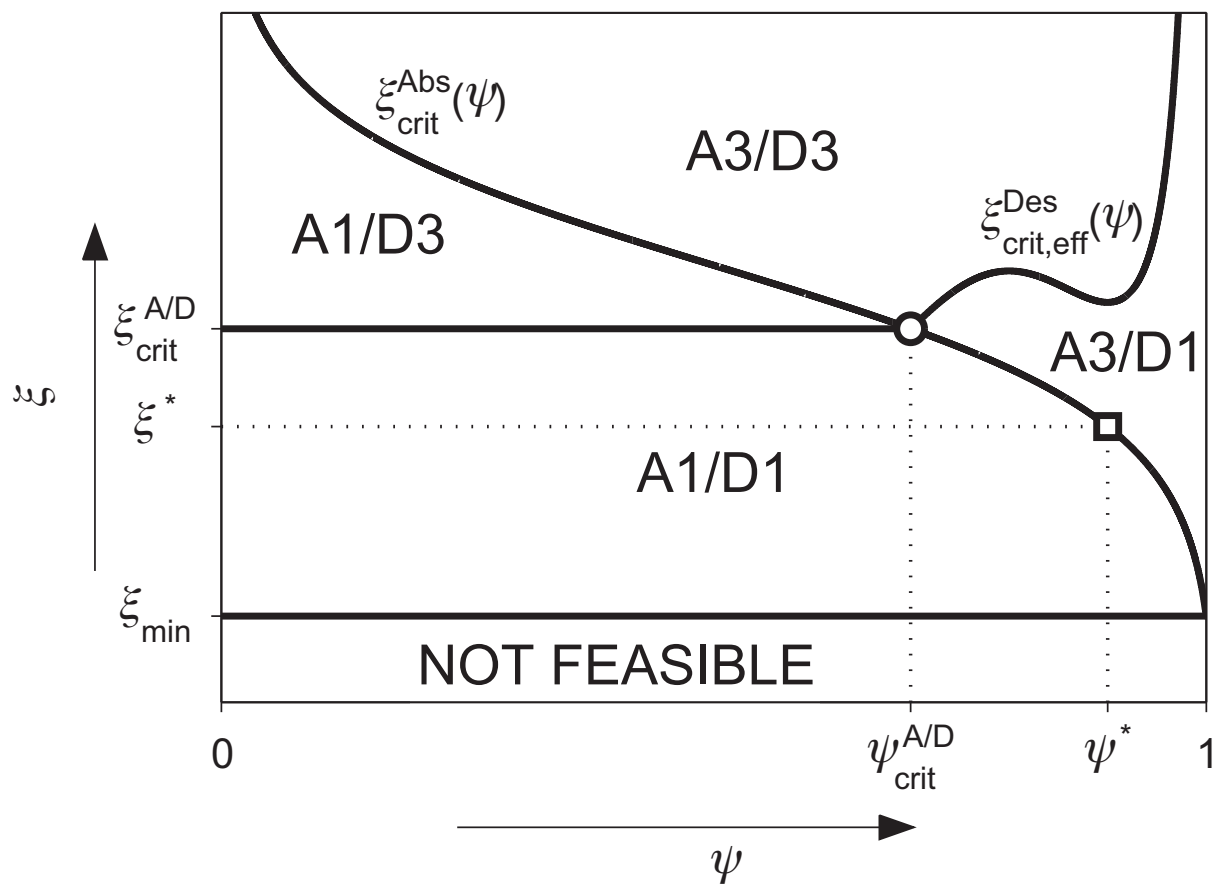

Figure 9: Overview of the topology of the solutions of the absorber / desorber model under the NoVa assumptions. There are four different regions which refer to combinations of different cases for the operation of absorber and desorber, see Figure 3 and Figure 4. The position of the global optimal point ( $\square$ ) and the absorber / desorber critical point ( $\bigcirc)$ are indicated. 
Table 2: Characterization of three solvents for $\mathrm{CO}_{2}$ capture based on the three coordinates of the global optimum point determined with the NoVa method. The scenario is BC2.

\begin{tabular}{cccc}
\hline Solvent & $q_{\mathrm{min}}^{\mathrm{Reb}^{*}} / \mathrm{GJ} \cdot \mathrm{t}_{\mathrm{CO}_{2}}^{-1}$ & $\xi^{*} / \mathrm{kg} \cdot \mathrm{kg}^{-1}$ & $1-\psi^{*}$ \\
\hline MEA30 & 3.43 & 3.7 & $2 \cdot 10^{-3}$ \\
MDEA30 & 2.45 & 4.3 & $3 \cdot 10^{-2}$ \\
BuTAD30 & 2.26 & 2.6 & $5 \cdot 10^{-11}$ \\
\hline
\end{tabular}

of $1-\psi^{\star}$ would be an interesting assessment criterion. The orders of magnitudes of $1-\psi^{\star}$ for the three solvents differ strongly, but the numbers for $\psi^{\star}$ are all very close to 1 . This means that for typical specifications of $\psi$, the number for $q_{\min }^{\mathrm{Reb}}$ does not depend on $\psi$ and is equal to $q_{\mathrm{min}}^{\mathrm{Reb} *}$. As a consequence, also $\xi^{*}$ does not depend on $\psi$ for the technically interesting choices of $\psi$. This result highlights the importance of $q_{\mathrm{min}}^{\mathrm{Reb}}$ and $\xi^{*}$ for assessing solvents. The plots $q^{\operatorname{Reb}}(\xi)$ for MEA30, MDEA30 and BuTAD30 in scenario BC2 are presented in Figure 10 .

\section{Conclusions}

The short-cut method of Notz et al. $\stackrel{1}{ }$ for assessing solvents for gas purification in absorption / desorption processes was developed further in the present work. The enhanced short-cut method is named NoVa. Compared to the method of Notz, the NoVa method omits the discretization of the equilibrium curves. Thus, it is easier to apply. Moreover, the NoVa method is based on the assumption of an infinite number of stages both in the absorber and desorber. Thus it is conceptually more straightforward. The NoVa method gives similar results as the method of Notz et al. $\frac{1}{1}$ is simpler to handle, and enables new insights in the process. Namely, we show that a threshold value of the removal rate $\psi^{\star}$ exists. The minimum specific regeneration energy does not depend on the removal rate below this threshold value when an infinite number of stages is assumed. The number obtained for the minimum specific regeneration energy in that region $q_{\min }^{\mathrm{Reb*}}$ is the global optimum for a given separation task and solvent and hence, useful for comparing solvents. Also the ratio of the liquid and the gas mass flowrate $(L / G)^{*}$ in that point is useful for comparing solvents. In principle, also the threshold value $\psi^{\star}$ can be used for assessing solvents, even though it is typically 


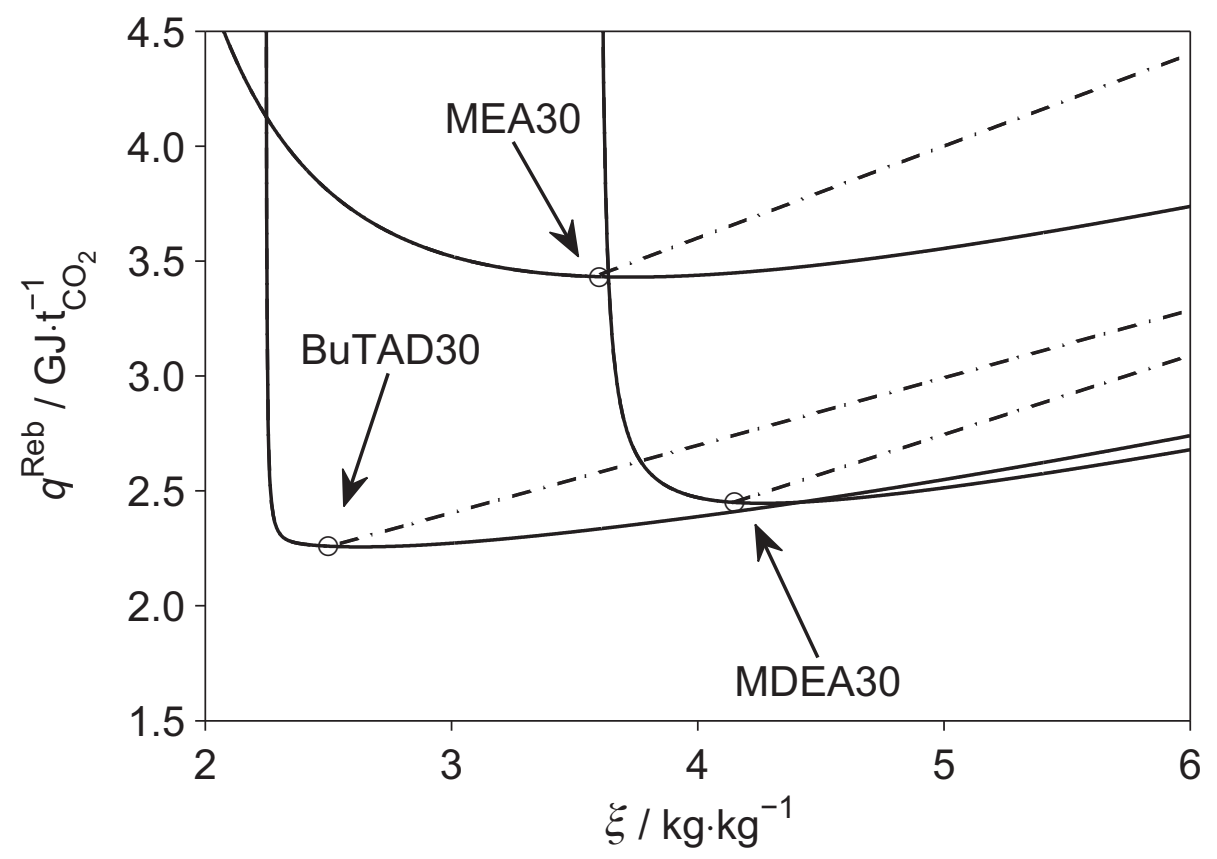

Figure 10: Specific regeneration energy of different solvents for scenario BC2 as a function of $\xi$ calculated with the NoVa method. (O) indicates the position of the specific regeneration energy optimum $q_{\mathrm{min}}^{\mathrm{Reb} *}$. The dash-dotted lines represent the second branch of the curves for $\psi^{*}$. The continuous lines represent the envelope for $\psi \rightarrow 0$, cf. Figure 8 , 
well above the numbers which are usually specified for the removal rate in industrial applications.

The NoVa method was applied here for assessing three solvents for scrubbing $\mathrm{CO}_{2}$ : MEA30, MDEA30, which are well known, as well as a new solvent BuTAD30. The latter was found to be interesting. In the screening, the results of NoVa on the energy requirement and the stream table of the process must be combined with additional considerations, regarding absorption kinetics, demixing, solid precipitation, toxicity, price and availability, etc.

Furthermore, a new equation for correlating gas solubility isotherms, the SolSOFT equation, is introduced, which is simple and well-behaved when used for extrapolations. It is shown that it can also be used successfully for calculating enthalpies of absorption from gas solubility data.

\section{Acknowledgments}

We gratefully acknowledge the financial support of this work by Federal Ministry for Economic Affairs and Energy of the Federal Republic of Germany under the project 03ET1098A-C, by Evonik Industries AG, Germany and by thyssenkrupp Industrial Solutions AG, Germany. 


\section{Appendix A. Physico-chemical background of SolSOFT}

The loading $\alpha_{\mathrm{G}}$ can be calculated from the overall molality of the gas $\mathrm{G}$ and the amine A with Eq. (31).

$$
\alpha_{\mathrm{G}}=\bar{m}_{\mathrm{G}} / \bar{m}_{\mathrm{A}}
$$

The molality $\bar{m}$ refers to water as solvent. In this discussion, the plain $\bar{m}_{i}$ denotes the molality of the apparent species $i$, while the starred $\bar{m}_{i}^{*}$ denotes the molality of the true species $i$.

For physical absorption, Henry's law can be written as:

$$
K_{\mathrm{H}} \cdot \bar{m}_{\mathrm{G}}^{*}=p_{\mathrm{G}}
$$

Combining this with the first term of the right hand side of MT-Eq. (27) and assuming $m=1$ yields:

$$
K_{\mathrm{p}}=K_{\mathrm{H}} \cdot \bar{m}_{\mathrm{A}}
$$

Hence, the parameter $K_{\mathrm{p}}$ can be considered as a modified Henry's law constant. For the empirical parameter $m$, the value $m=1$ is a good default choice. A chemical contribution is added assuming the stoichiometry:

$$
\mathrm{A}+\mathrm{nG} \rightleftharpoons \mathrm{AG}_{\mathrm{n}}
$$

For an ideal system the chemical equilibrium constant of the reaction can be written as shown in Eq. (35) where $\bar{m}_{0}=1 \mathrm{~mol} \cdot \mathrm{kg}^{-1}$.

$$
K_{\overline{\mathrm{m}}}=\frac{\bar{m}_{\mathrm{AG}}^{*} \cdot\left(\bar{m}_{0}\right)^{n}}{\bar{m}_{\mathrm{A}}^{*} \cdot\left(\bar{m}_{\mathrm{G}}^{*}\right)^{n}}
$$

From the mass balances of G (cf. Eq. (36)) and of A (cf. Eq. (37)), it is found that the number $n$ 
in MT-Eq. (27) is the stoechiometric factor in Eq. (34) and that $K_{\mathrm{c}}$ from MT-Eq. (27) can be related to $K_{\overline{\mathrm{m}}}$ and $K_{\mathrm{H}}$ by Eq. (38).

$$
\begin{gathered}
\bar{m}_{\mathrm{G}}=\bar{m}_{\mathrm{G}}^{*}+n \cdot \bar{m}_{\mathrm{AG}}^{*} \\
\bar{m}_{\mathrm{A}}=\bar{m}_{\mathrm{A}}^{*}+\bar{m}_{\mathrm{AG}}^{*} \\
K_{\mathrm{c}}=K_{\mathrm{H}} \cdot\left(K_{\overline{\mathrm{m}}}\right)^{-\frac{1}{n}} \cdot \bar{m}_{0}
\end{gathered}
$$

If the chemical term dominates, which is usually the case for reactive solvents at low gas partial pressures, the physical term can be neglected. Eq. (39) presents a variant of SolSOFT with only a chemical term. Eq. (39), which preserves the favorable mathematical properties of SolSOFT, possess additionally an explicitly defined inverse $p_{\mathrm{G}}\left(\alpha_{\mathrm{G}}\right)$. In Eq. (39) $m^{*}$ is a dimensionless adjustable parameter.

$$
\alpha_{\mathrm{G}}=m^{*} \cdot n \cdot \frac{\left(p_{\mathrm{G}} / K_{\mathrm{c}}\right)^{n}}{1+\left(p_{\mathrm{G}} / K_{\mathrm{c}}\right)^{n}}
$$

In the limiting case $p_{\mathrm{G}} \rightarrow 0$ (or equivalently $\alpha_{\mathrm{G}} \rightarrow 0$ ) Eq. (27) with $m=1$ yields $p_{\mathrm{G}} / \alpha_{\mathrm{G}}=K_{\mathrm{p}}$, i.e. a constant finite value. However, for any $m<1$ that limit will go to zero whereas for all $m>1$ it will go to infinity. Nevertheless, good fits of data at both high and low concentrations are achieved with Eq. (27) as shown in this work. Should problems occur, they can be circumvented by the following empirical extension of Eq. (27):

$$
\begin{gathered}
\alpha_{\mathrm{G}}=F \cdot\left(p_{\mathrm{G}} / K_{\mathrm{p}}\right)+n \cdot \frac{\left(p_{\mathrm{G}} / K_{\mathrm{c}}\right)^{n}}{1+\left(p_{\mathrm{G}} / K_{\mathrm{c}}\right)^{n}} \\
F=\frac{\left(p_{\mathrm{G}} / K_{\mathrm{p}}\right)^{m}+\varphi}{\left(p_{\mathrm{G}} / K_{\mathrm{p}}\right)+\varphi}
\end{gathered}
$$


and assigning a small constant value fixed to $\varphi$. This fixed value can be chosen in a preliminary study, i.e. it is rather a numerical parameter than a typical fitting parameter - even though it could be used also in that way. After assigning a fixed value to $\varphi$, the limit of $p_{\mathrm{G}} / \alpha_{\mathrm{G}}$ for $p_{\mathrm{G}} \rightarrow 0$ (or $\alpha_{\mathrm{G}} \rightarrow 0$ ) obtained from Eq. (40) is always $K_{\mathrm{p}}$.

\section{Appendix B. Enthalpy of absorption from SolSOFT}

The differential enthalpy of absorption of the gas $\mathrm{G}$ in the solvent $\mathrm{S} \Delta_{\mathrm{abs}}^{\mathrm{diff}} h_{\mathrm{G}}^{\mathrm{S}}$ can be determined from temperature-dependent SolSOFT equation for strictly positive loadings by Eq. (42). Here $A_{\mathrm{p}}^{\text {diff }}$ and $A_{\mathrm{c}}^{\text {diff }}$ are defined by Eq. (43) and Eq. (44).

$$
\begin{gathered}
\Delta_{\mathrm{abs}}^{\mathrm{diff}} h_{\mathrm{G}}^{\mathrm{S}}\left(p_{\mathrm{G}}, T\right)=\mathrm{R} \cdot \frac{b_{\mathrm{p}} \cdot A_{\mathrm{p}}^{\text {diff }}+b_{\mathrm{c}} \cdot A_{\mathrm{c}}^{\text {diff }}}{A_{\mathrm{p}}^{\text {diff }}+A_{\mathrm{c}}^{\text {diff }}} \\
A_{\mathrm{p}}^{\text {diff }}=m \cdot\left(p_{\mathrm{G}} / K_{\mathrm{p}}\right)^{m} \\
A_{\mathrm{c}}^{\text {diff }}=n^{2} \cdot \frac{\left(p_{\mathrm{G}} / K_{\mathrm{c}}\right)^{n}}{\left[1+\left(p_{\mathrm{G}} / K_{\mathrm{c}}\right)^{n}\right]^{2}}
\end{gathered}
$$

The integral enthalpy of absorption, which is the caloric effect associated to the absorption of gas into a completely unloaded solvent up to a certain gas loading, can be used as an average value of the differential enthalpy of absorption between 0 and the partial pressure $p_{\mathrm{G}}$ (cf. Eq. (45)).

$$
\Delta_{\mathrm{abs}}^{\mathrm{int}} h_{\mathrm{G}}^{\mathrm{S}}\left(p_{\mathrm{G}}, T\right)=\frac{1}{\alpha_{\mathrm{G}}\left(p_{\mathrm{G}}, T\right)} \cdot \int_{0}^{\alpha_{\mathrm{G}}\left(p_{\mathrm{G}}, T\right)} \Delta_{\mathrm{abs}}^{\mathrm{diff}} h_{\mathrm{G}}^{\mathrm{S}}(\alpha, T) \mathrm{d} \alpha
$$

In the case of the SolSOFT equation, the definite integral in Eq. (45) can be written as an algebraic expression and the integral enthalpy of absorption can be explicitly given as Eq. (46). 
Here $A_{\mathrm{p}}^{\mathrm{int}}$ and $A_{\mathrm{c}}^{\mathrm{int}}$ are defined by Eq. (47) and Eq. (48).

$$
\begin{gathered}
\Delta_{\mathrm{abs}}^{\mathrm{int}} h_{\mathrm{G}}^{\mathrm{S}}\left(p_{\mathrm{G}}, T\right)=\mathrm{R} \cdot \frac{b_{\mathrm{p}} \cdot A_{\mathrm{p}}^{\mathrm{int}}+b_{\mathrm{c}} \cdot A_{\mathrm{c}}^{\mathrm{int}}}{A_{\mathrm{p}}^{\mathrm{int}}+A_{\mathrm{c}}^{\mathrm{int}}} \\
A_{\mathrm{p}}^{\mathrm{int}}=\left(p_{\mathrm{G}} / K_{\mathrm{p}}\right)^{m} \\
A_{\mathrm{c}}^{\mathrm{int}}=n \cdot \frac{\left(p_{\mathrm{G}} / K_{\mathrm{c}}\right)^{n}}{1+\left(p_{\mathrm{G}} / K_{\mathrm{c}}\right)^{n}}
\end{gathered}
$$

Information on the behavior of the enthalpy of absorption calculated from the SolSOFT equation at zero loading and derivations of Eq. (42) and Eq. (46) are presented in Supplementary Information $\mathrm{C}$.

\section{Appendix C. Numerical aspects}

With one exception, the defining equations of the mathematical model of NoVa are explicit in the unknown variables. The exception is the nonlinear system, that defines the case D1 (cf. MTEq. (16) and MT-Eq. (21)). This system of equations does not possess an analytical solution.

For a numerical solution, these two equations can be coupled to yield a single equation from which $p_{\mathrm{CO}_{2}}^{\text {Des,pinch }}$ can be found. That equation is Eq. (49).

$$
X_{\mathrm{CO}_{2}}^{\mathrm{Lean}}=X_{\mathrm{CO}_{2}}^{\text {Des,eq }}\left(p_{\mathrm{CO}_{2}}^{\text {Des,pinch }}\right)-\left.p_{\mathrm{CO}_{2}}^{\text {Des,pinch }} \cdot \frac{\mathrm{d} X_{\mathrm{CO}_{2}}^{\text {Des,eq }}\left(p_{\mathrm{CO}_{2}}\right)}{\mathrm{d} p_{\mathrm{CO}_{2}}}\right|_{p_{\mathrm{CO}_{2}}=p_{\mathrm{CO}}^{\text {Des,pinch }}}
$$

Eq. (49) possess an unique physically meaningful (positive) solution. $\theta^{\text {Des }}$ is calculated subsequently with MT-Eq. (16).

The SolSOFT equation is explicit in the loading $\alpha_{\mathrm{CO}_{2}}$ and it generally does not possess an inverse defined by an algebraic expression. The partial pressure at equilibrium $p_{\mathrm{CO}_{2}}$, corresponding to an arbitrary loading $\alpha_{\mathrm{CO}_{2}}$, can therefore be calculated only numerically. 
For the cases solved in this work, fixed-point iteration procedures were employed to solve Eq. (49) and to calculate the partial pressure from a known loading.

The NoVa method was implemented in MATLAB Version 7.11.0.584. and in Microsoft Excel 2010. The Excel file can be downloaded under a free academic license from the homepage http://thermo.mv.uni-kl.de/links/. 


\section{References}

[1] R. Notz, I. Tönnies, H. P. Mangalapally, S. Hoch, H. Hasse, A short-cut method for assessing absorbents for post-combustion carbon dioxide capture, International Journal of Greenhouse Gas Control 5 (2011) 413 - 421.

[2] H. Kim, S. J. Hwang, K. S. Lee, Novel shortcut estimation method for regeneration energy of amine solvents in an absorption-based carbon capture process, Environ. Sci. Technol. 49 (2015) $1478-1485$.

[3] K. Jiang, K. Li, G. Puxty, H. Yu, P. H. M. Feron, Information derivation from vapor - liquid equilibria data: A simple shortcut to evaluate the energy performance in an amine-based postcombustion $\mathrm{CO}_{2}$ capture, Environ. Sci. Technol. 52 (2018) 10893 - 10901.

[4] H. K. Knuutila, R. Rennemo, A. F. Ciftja, New solvent blends for post-combustion $\mathrm{CO}_{2}$ capture, Green Energy \& Environment (2019) Article in press.

[5] O. Spuhl, H. Garcia, G. Sieder, R. Notz, Comparison and limitation of different evaluation methods for novel PCC solvents, Energy Procedia 4 (2011) 51 - 58.

[6] O. Ryll, S. Blagov, H. Hasse, $\infty / \infty$ - analysis of homogeneous distillation processes, Chem. Eng. Sci. 84 (2012) $315-332$.

[7] O. Ryll, S. Blagov, H. Hasse, $\infty / \infty$ - analysis of heterogeneous distillation processes, Chem. Eng. Sci. 104 (2013) $374-388$.

[8] O. Ryll, S. Blagov, H. Hasse, Thermodynamic analysis of reaction-distillation processes based on piecewise linear models, Chem. Eng. Sci. 109 (2014) 284 - 295.

[9] J. Burger, M. Kaul, H. Hasse, Slope curve method for the analysis of separations in extraction columns of infinite height, Chem. Eng. Sci. 143 (2016) 105 - 113. 
[10] D. Vasiliu, A. Yazdani, N. McCann, M. Irfan, R. Schneider, J. Rolker, G. Maurer, E. von Harbou, H. Hasse, Thermodynamic study of a complex system for carbon capture: Butyltriacetonediamine + water + carbon dioxide, J. Chem. Eng. Data 61 (11) (2016) 3814-3826.

[11] E. Kessler, L. Ninni Schäfer, B. Willy, R. Schneider, M. Irfan, J. Rolker, E. von Harbou, H. Hasse, Structure-property relationships for new amines for reactive $\mathrm{CO}_{2}$ absorption, Chem. Eng. Trans. 69 (2018) 109 - 114.

[12] P. M. Mathias, J. P. O'Connell, The Gibbs-Helmholtz equation and the thermodynamic consistency of chemical absorption data, Ind. Eng. Chem. Res. 51 (2012) 5090 - 5097.

[13] M. Wagner, I. von Harbou, J.-I. Kim, I. Ermatchkova, G. Maurer, H. Hasse, Solubility of carbon dioxide in aqueous solutions of monoethanolamine in the low and high gas loading regions, J. Chem. Eng. Data 58 (2013) 883 - 895.

[14] V. Ermatchkov, G. Maurer, Solubility of carbon dioxide in aqueous solutions of $n$ methyldiethanolamine and piperazine: Prediction and correlation, Fluid Ph. Equilibria 302 (2011) $338-346$.

[15] I. Kim, H. F. Svendsen, Heat of absorption of carbon dioxide $\left(\mathrm{CO}_{2}\right)$ in monoethanolamine (MEA) and 2-(aminoethyl)ethanolamine (AEEA) solutions, Ind. Eng. Chem. Res. 46 (2007) $5803-5809$.

[16] I. von Harbou, Post-combustion carbon capture by reactive absorption using aqueous amine solutions - experiments, modeling and simulation, 152 - 157, Laboratory of Engineering Thermodynamics - Technical University of Kaiserslautern (2013).

[17] H. P. Mangalapally, H. Hasse, Pilot plant study of post-combustion carbon dioxide capture by reactive absorption: Methodology, comparison of different structured packings, and comprehensive results for monoethanolamine, Chem. Eng. Res. Des. 89 (2011) 1216 - 1228. 
[18] H. P. Mangalapally, H. Hasse, Pilot plant experiments for post combustion carbon dioxide capture by reactive absorption with novel solvents, Energy Procedia 4 (2011) 1 - 8. 